\title{
A global take on legal aspects of fantasy sports
}

\author{
Johan Lindholm ${ }^{1}$
}

Published online: 28 September 2021

(c) T.M.C. Asser Instituut 2021

Although it has been around for more than half a century, fantasy sports remain for many a relatively new way to engage with sports. Fantasy sports is a game where players create virtual teams by selecting real athletes and score points depending on how those athletes perform in realworld competitions according to a set of statistical variables and pre-determined rules. It is a game that rewards players' ability to accurately predict sporting outcomes while making strategic choices within the limits of a particular game. Beyond these basic shared characteristics, fantasy sports vary greatly in design: it can be based on any real sport, play out over a single day or multiple seasons, provide winners with bragging rights or substantial cash prices, and have a near infinite variation of scoring rules.

Fantasy sports originated in North America. Before the widespread use of computers and the internet, fantasy leagues were formed by groups of people who knew each other in real life and the design of the games were by necessity simple, favoring statistically straight-forward sports. ${ }^{1}$ Early fantasy sports were decentralized and anyone with a few friends, a little time and patience, pen and paper, and a subscription to a daily newspaper with a decent sports section could play. Fantasy sports attained a more significant following in the 1980s in the United States after a group of New York journalists created and wrote about the Rotisserie
League Baseball. ${ }^{2}$ As fantasy sports began to attract more players, an industry catering to fantasy players started to form. This industry was initially relatively modest in size, focusing on selling printed information and guides.

Since these almost quaint beginnings, fantasy sports radically evolved and with it an industry that is today a growing multi-billion-dollar business. ${ }^{3}$ Particularly three factors help explain this development.

First, the impact of the internet on fantasy sports cannot be overstated. As an increasing number of people attained access to the internet at home in the 1990s, major U.S. businesses such as Yahoo, CBS, and ESPN introduced websitebased fantasy platforms. These platforms greatly simplified organizing and running fantasy leagues, while also providing fantasy sports-related news and tips. They thereby made fantasy sports increasingly attractive and accessible to large segments of sports fans. These websites often offer a way to play for free but may involve entry fees and cash prices and may also offer additional services for pay. The subsequent technological development, particularly the introduction of smartphones, mobile internet, and application-based fantasy sports, has helped fantasy sports grow further. Today, there are more than 60 million fantasy sports players in the United States alone.
Johan Lindholm

johan.lindholm@umu.se

1 Umeå University, Umeå, Sweden

\footnotetext{
${ }^{1}$ It is difficult to confirm, but the first fantasy sports league according to most sources was a 1950s fantasy golf league established by Wilfred Winkenbach, where participating players picked a team of PGA golfers. Winkenbach was also part-owner of a National Football League (NFL) franchise and is also credited with the creation of fantasy football.

2 The league was named after the restaurant where the first draft was held, La Rotisserie Française.

3 Arizton, Fantasy Sports Market - Global Outlook and Forecast 2021-2026. Available at https://www.arizton.com/market-reports/ fantasy-sports-market. Accessed August 19, 2021.
} 
Second, in the late 2000s, new operators such as DraftKings and FanDuel started offering a new, high-pace version of fantasy sports referred to as daily fantasy sports (DFS). Whereas fantasy games would traditionally play out over the course of an entire sporting season, DFS takes place in a single day. DFS players pay fees to play and for the chance to win potentially significant cash prices. In this manner, DFS has contributed to radically increasing the amount of money involved in fantasy sports.

Third, although North America still dominates global fantasy sport, the phenomenon is rapidly spreading to the rest of the world. Millions of people around the world now play fantasy games based on for example European soccer, such as Fantasy Premier League and Fantasy Bundesliga, or the Indian Premier League (IPL) in cricket. This development has opened up fantasy sports to new, large fan bases around the globe, further increasing fantasy sports's growth potential.

In this special issue of the International Sports Law Journal leading experts from around the world offer a broad and comparative perspective on legal issues related to fantasy sports. The contributions center around two main themes.

The first theme is intellectual property rights and the equitable distribution of profits. Like other games, fantasy sports are a (hopefully) fun social activity. It also adds a new dimension of interest to the sport and league on which the fantasy game is based and, as a result, can help raise interest in that sport. ${ }^{4}$ In this way, a flourishing fantasy community can be positive for a sports league, including financially advantageous. However, the fantasy sports industry is also making significant profits for itself based on sports data, specifically statistical information on how individual athletes performed on teams in sporting events. Consequently, the competing athletes, the teams that they are associated with, and the organizers of the sporting events can and frequently do claim a legal right to that data and, by extension, a share in the fantasy sports industry's profits.

The second theme is the regulation of fantasy sports, particularly as a form of gambling. Whereas minor elements of gambling have been part of fantasy sports since the beginning, the introduction of DFS has arguably turned fantasy sports into a major gambling industry. This has prompted the question of whether fantasy sports, or at least DFS, falls under the same regulations as other forms of gambling or should be regulated in the same manner. At the same time, fantasy sports differ in some regards from more traditional forms of gambling in a way that complicates regulation.

We are extremely grateful to the participating authors and hope that our readership will enjoy this deep dive into this somewhat niche but increasingly significant aspect of sports.

Publisher's Note Springer Nature remains neutral with regard to jurisdictional claims in published maps and institutional affiliations.

\footnotetext{
4 Although, as anyone who plays fantasy sports well knows, it is a curious and somewhat uncomfortable form of attention that comes with rooting for individual player performances rather than match outcomes.
} 